\title{
ON TOTAL FROBENIUS-SCHUR INDICATORS
}

\author{
GONGXIANG LIU AND SIU-HUNG NG
}

\begin{abstract}
We define total Frobenius-Schur indicator for each object in a spherical fusion category $\mathcal{C}$ as a certain canonical sum of its higher indicators. The total indicators are invariants of spherical fusion categories. If $\mathcal{C}$ is the representation category of a semisimple quasi-Hopf algebra $H$, we prove that the total indicators are non-negative integers which satisfy a certain divisibility condition. In addition, if $H$ is a Hopf algebra, then all the total indicators are positive. Consequently, the positivity of total indicators is a necessary condition for a quasi-Hopf algebra being gauge equivalent to a Hopf algebra. Certain twisted quantum doubles of finite groups and some examples of Tambara-Yamagami categories are discussed for the sufficiency of this positivity condition.
\end{abstract}

\section{INTRODUCTION}

The representation category $\operatorname{Rep}(H)$ of a Hopf algebra $H$ is certainly important to the understanding of the algebraic structure of $H$. The monoidal structure of $\operatorname{Rep}(H)$ has also been playing important roles in other areas of mathematics and physics. For instance, the quantum invariants of knots, links or 3-manifolds constructed from certain Hopf algebras are actually determined by the monoidal structures of their representation categories [33].

Quasi-Hopf algebras are generalizations of Hopf algebras whose representation categories are also monoidal categories. Two quasi-Hopf algebras are said to be gauge equivalent if their representation categories are monoidally equivalent. For any finite-dimensional quasi-Hopf algebra $K$ over $\mathbb{C}$, one can obtain another quasiHopf algebra $K^{F}$ by twisting $K$ with a gauge transformation $F \in K \otimes K$ [14], but $K^{F}$ and $K$ are gauge equivalent. In general, two finite-dimensional quasiHopf algebras $K$ and $H$ are gauge equivalent if, and only if, there exists a gauge transformation $F \in K \otimes K$ such that $K^{F}$ and $H$ are isomorphic as quasi-bialgebras (cf. $[7,25]$ ). However, it is generally difficult to decide the gauge equivalence of two finite-dimensional quasi-Hopf algebras if their Grothendieck rings happen to be isomorphic.

For any finite group $G$, and a normalized 3-cocycle $\omega$ on $G$ with values in $\mathbb{C}^{\times}$, one can construct a semisimple quasi-Hopf algebra $D^{\omega}(G)$, called a twisted quantum double of $G$ [5]. Dijkgraaf, Pasquier and Roche have asked the question whether $D^{\omega}(G)$ is gauge equivalent to some Hopf algebra in [5]. One can even ask a more general question: How can one determine whether a given finite-dimensional quasiHopf algebra $K$ over $\mathbb{C}$ is gauge equivalent to some Hopf algebra? In view of the reconstruction theorem, the question is equivalent to ask for the existence of a

The first author was partially supported by the NSF grant number 11071111 and the second author was partially supported by NSF grant number DMS1001566. 
fibration, i.e. a $\mathbb{C}$-linear, faithful and exact monoidal functor $\mathcal{F}: \operatorname{Rep}(K) \rightarrow \operatorname{Vect}_{\mathbb{C}}$, where Vect $\mathbb{C}$ denotes the category of finite-dimensional $\mathbb{C}$-linear spaces.

This simply stated question is generally difficult to answer. One can work out the simplest example $D^{\omega}\left(\mathbb{Z}_{2}\right)$, where $\omega$ is a non-trivial 3-cocycle of $\mathbb{Z}_{2}$. It is not completely obvious that $D^{\omega}\left(\mathbb{Z}_{2}\right)$ is not gauge equivalent to any Hopf algebra [23].

The $n$-th Frobenius-Schur (FS) indicator $\nu_{n}(V)$ of a representation $V$ of a finite group was introduced for more than a century. It has been generalized to the representations of a semisimple (quasi-)Hopf algebra [16, 24], to the primary fields of a rational conformal field theory [3], and more generally to the objects of a pivotal categories [27]. These indicators are preserved by equivalences of pivotal category [loc. cit.]. The arithmetical properties of the FS indicators also encrypt the structure of the underlying tensor categories as well as the quasi-Hopf algebras. For instance, the indicators of any complex representation of a finite group are integers.

If $V$ is an object of a spherical fusion category $\mathcal{C}$ over $\mathbb{C}$, it has been shown that the sequence $\nu(V):=\left\{\nu_{n}(V)\right\}_{n \in \mathbb{N}}$ of FS indicators is a periodic sequence of cyclotomic integers. Moreover, there exists a global period $N$ for all the higher indicator sequences [26]. This global period, denoted by $\operatorname{FSexp}(\mathcal{C})$, is called the Frobenius-Schur exponent of $\mathcal{C}$, and it is also an invariant of spherical fusion categories. The representation category $\operatorname{Rep}(H)$ of a semisimple quasi-Hopf algebra over $\mathbb{C}$ is a spherical fusion category [8], and we simply denote the Frobenius-Schur exponent of $\operatorname{Rep}(H)$ by $\mathrm{FSexp}(H)$.

The FS indicators of a representation of semisimple Hopf algebra are not necessarily integers (see [13] for example). However, if we consider the total indicator $\bar{\nu}(V)$ defined for any object $V$ in a spherical fusion category $\mathcal{C}$ by

$$
\bar{\nu}(V):=\sum_{n=1}^{N} \nu_{n}(V)
$$

where $N=\mathrm{FS} \exp (\mathcal{C})$, then we have the following integrality and divisibility theorem for quasi-Hopf algebras.

Theorem A. Let $H$ be a semisimple quasi-Hopf algebra over $\mathbb{C}$. For any finitedimensional $H$-module $V, \bar{\nu}(V)$ is a non-negative integer which satisfies the divisibility

$$
\operatorname{FSexp}(H) \mid(\operatorname{dim} H) \cdot \bar{\nu}(V) .
$$

In addition, for semisimple Hopf algebras, we have obtained the positivity of total indicators:

Theorem B. Let $H$ be a semisimple Hopf algebra $\mathbb{C}$. Then, $\bar{\nu}(V) \geq \frac{N \operatorname{dim} V}{\operatorname{dim} H}$ for all finite-dimensional $H$-modules $V$, where $N=\operatorname{FS} \operatorname{Sexp}(H)$. In particular, $\bar{\nu}(V)>0$ for any non-zero $H$-module $V$.

Since $\operatorname{FSexp}(H)=\exp (H)$ when $H$ is a semisimple Hopf algebra, Theorem A provides another perspective for a conjecture of Kashina [11, 12]: $\exp (H)$ divides $\operatorname{dim} H$ for any semisimple Hopf algebra $H$ over $\mathbb{C}$. Moreover, Theorem B yields a necessary condition for a semisimple quasi-Hopf algebra being gauge equivalent to a Hopf algebra. However, this necessary condition may not be sufficient in general. There are integral Tambara-Yamagami categories which has no fibration but their total indicators are all positive. Nevertheless, the positivity of total indicators is a 
necessary and sufficient for abelian twisted quantum doubles being gauge equivalent to Hopf algebras.

The organization of the paper is as follows: In Section 2, we introduce the definition of total Frobenius-Schur indicators and prove Theorem A. Section 3 is devoted to the proof of Theorem B. In Section 4, we consider twisted quantum doubles of $D^{\omega}(G)$ of a finite abelian group $G$, and show that if $D^{\omega}(G)$ is commutative, then $D^{\omega}(G)$ is gauge equivalent to a Hopf algebra if, and only if, $\bar{\nu}(V)>0$ for all $V \in \operatorname{Rep}\left(D^{\omega}(G)\right)$. This provides an answer to a question of Dijkgraaf, Pasquier and Roche [5, p69]. In Section 5, we compute the total indicators for some integral Tambara-Yamagami fusion categories, and we characterize those admitting a fibration in terms of total indicators. As a consequence, semisimple quasi-Hopf algebras with positive total indicators but not gauge equivalent to any Hopf algebra are found.

\section{Total FrobeniUs-Schur Indicators}

Throughout this paper, unless stated otherwise, we will work over the field $\mathbb{C}$ of complex numbers; every monoidal category $\mathcal{C}$ in this paper is assumed to be $\mathbb{C}$-linear abelian with finite-dimensional Hom-spaces over $\mathbb{C}$ and a strict simple unit object $\mathbf{1}_{\mathcal{C}}$. All (quasi-)Hopf algebras are assumed to be semisimple and finite-dimensional over $\mathbb{C}$. We denote by $\operatorname{Rep}(H)$ the $\mathbb{C}$-linear monoidal category of finite-dimensional representations of a quasi-Hopf algebra $H$. The unit object of $\operatorname{Rep}(H)$, simply denoted by $\mathbf{1}_{H}$, is the $H$-module $\mathbb{C}$ equipped with the trivial $H$-action.

In this section, we collect some conventions, and recall some basic definitions and facts for the discussions in the remaining sections. The readers may refer to $[2,8,21]$ for the basic theory of tensor categories and $[16,13,27,25]$ for FrobeniusSchur indicators. The aim of this section is to introduce the definition of total Frobenius-Schur indicators (abbr. total indicators), and to prove Theorem A.

Let $\mathcal{C}$ be a left rigid monoidal category with tensor product $\otimes$. The left dual of $V \in \mathcal{C}$ is a triple $\left(V^{*}, \mathrm{db}, \mathrm{ev}\right)$ in which $V^{*} \in \mathcal{C}$, and $\mathrm{db}: \mathbf{1} \rightarrow V \otimes V^{*}$ and ev : $V^{*} \otimes V \rightarrow \mathbf{1}$ are respectively the associated dual basis and evaluation morphisms of the left dual. The left duality on $\mathcal{C}$ can be extended to a monoidal equivalence $(-)^{*}: \mathcal{C}^{\text {op }} \rightarrow \mathcal{C}$ and hence $(-)^{* *}: \mathcal{C} \rightarrow \mathcal{C}$ defines a monoidal equivalence. A pivotal structure on $\mathcal{C}$ is an isomorphism $j: \mathrm{Id} \rightarrow(-)^{* *}$ of monoidal functors, and the pair $(\mathcal{C}, j)$ is a called a pivotal category. We will simply say that $\mathcal{C}$ is a pivotal category when the pivotal structure is understood without ambiguity.

In a pivotal category $(\mathcal{C}, j)$, one can define the left and right pivotal traces for any endomorphism $f: V \rightarrow V$ in $\mathcal{C}$ as

$$
\begin{aligned}
& \underline{\operatorname{ptr}}^{r}(f):=\left(\mathbf{1} \stackrel{\mathrm{db}}{\longrightarrow} V \otimes V^{*} \stackrel{f \otimes V^{*}}{\longrightarrow} V \otimes V^{*} \stackrel{j_{V} \otimes V^{*}}{\longrightarrow} V^{* *} \otimes V^{*} \stackrel{\mathrm{ev}}{\longrightarrow} \mathbf{1}\right), \\
& \underline{\operatorname{ptr}^{\ell}}(f):=\left(\mathbf{1} \stackrel{\mathrm{db}}{\longrightarrow} V^{*} \otimes V^{* *} \stackrel{V^{*} \otimes j_{V}^{-1}}{\longrightarrow} V^{*} \otimes V \stackrel{V^{*} \otimes f}{\longrightarrow} V^{*} \otimes V \stackrel{\mathrm{ev}}{\longrightarrow} \mathbf{1}\right)
\end{aligned}
$$

respectively. Note that these traces are scalars as $\mathbf{1}$ is simple. A spherical category is a pivotal category $\mathcal{C}$ in which $\underline{\operatorname{ptr}}^{r}(f)=\underline{\operatorname{ptr}}^{\ell}(f)$ for all endomorphisms $f \in \mathcal{C}$. In this case, we simply write $\underline{\mathrm{ptr}}$ for the functions $\underline{\operatorname{ptr}^{r}}$ as well as $\underline{\operatorname{ptr}}{ }^{\ell}$, and $d(V):=\underline{\operatorname{ptr}}\left(\operatorname{id}_{V}\right)$ is called the pivotal dimension of $V$. In addition, if $\mathcal{C}$ is semisimple with finitely many simple objects up to isomorphism, then $\mathcal{C}$ is called a spherical fusion category (cf. [8] for more details on fusion categories). In this case, $d(V)$ is a non-zero real 
number (cf. [8]), and the global dimension $\operatorname{dim} \mathcal{C}$ of $\mathcal{C}$ is defined as

$$
\operatorname{dim} \mathcal{C}=\sum_{V \in \operatorname{Irr}(\mathcal{C})} d(V)^{2}
$$

where $\operatorname{Irr}(\mathcal{C})$ denotes a complete set of non-isomorphic simple objects of $\mathcal{C}$.

By Müger [22], the center $\mathcal{Z}(\mathcal{C})$ is a modular tensor category. In particular, the associated twist (or ribbon structure) $\theta$ has finite order $[2,34]$. Moreover, the forgetful functor $F: \mathcal{Z}(\mathcal{C}) \rightarrow \mathcal{C}$ admits a two-sided adjoint $K: \mathcal{C} \rightarrow \mathcal{Z}(\mathcal{C})$.

Let $V$ be an object in a pivotal category $(\mathcal{C}, j)$ with associativity isomorphism $\Phi$. We write $V^{\otimes n}$ for the $n$-fold tensor power of $V \in \mathcal{C}$ with rightmost parentheses. By coherence theorem, there is a unique isomorphism

$$
\Phi^{(n)}: V^{\otimes(n-1)} \otimes V \rightarrow V^{\otimes n}
$$

which is a composition of tensor products of id, $\Phi$ and $\Phi^{-1}$. One can define the $\mathbb{C}$-linear operator $E_{V}^{(n)}: \mathcal{C}\left(\mathbf{1}, V^{\otimes n}\right) \rightarrow \mathcal{C}\left(\mathbf{1}, V^{\otimes n}\right)$ by setting

$$
\begin{aligned}
& E_{V}^{(n)}(f):=\left(1 \stackrel{\mathrm{db}}{\longrightarrow} V^{*} \otimes V^{* *} \stackrel{\left(V^{*} \otimes f\right) \otimes V^{* *}}{\longrightarrow}\left(V^{*} \otimes V^{\otimes n}\right) \otimes V^{* *} \stackrel{\Phi^{-1} \otimes j^{-1}}{\longrightarrow}\right. \\
&\left.\left(\left(V^{*} \otimes V\right) \otimes V^{\otimes(n-1)}\right) \otimes V \stackrel{\left(\mathrm{ev} \otimes V^{\otimes(n-1)}\right) \otimes V}{\longrightarrow} V^{\otimes(n-1)} \otimes V \stackrel{\Phi^{(n)}}{\longrightarrow} V^{\otimes n}\right) .
\end{aligned}
$$

Following [27, Sect. 3], the $n$-th Frobenius-Schur indicator $\nu_{n}(V)$ of $V$ is defined as the scalar

$$
\nu_{n}(V)=\operatorname{Tr}\left(E_{V}^{(n)}\right) .
$$

These indicators are proved to be invariant under pivotal equivalences, and $\nu_{n}(V)$ is a cyclotomic integer in $\mathbb{Q}\left(\zeta_{n}\right)$ where $\zeta_{n}$ is a primitive $n$-th root of unity (cf. [27]).

Since the antipode of a semisimple Hopf algebra $H$ is an involution [15], the representation category $\operatorname{Rep}(H)$ is a spherical fusion category in which the pivotal structure is the usual canonical isomorphism $j_{V}: V \rightarrow V^{* *}$ of finite-dimensional vector spaces. In this case, the pivotal dimension $d(V)$ of $V \in \operatorname{Rep}(H)$ is the ordinary dimension of $V$. More generally, for a semisimple quasi-Hopf algebra $H$, there is a unique (spherical) pivotal structure on $\operatorname{Rep}(H)$ such that $d(V)$ is the ordinary dimension of $V$ for all $V \in \operatorname{Rep}(H)$ [8]. Moreover, $\nu_{n}(V)$ can be expressed in terms of the associator, the quasi-antipode and the normalized integral $\Lambda$ of $H$ (cf. [25, Sect. 4]). When $H$ is a Hopf algebra, we recover the $n$-th Frobenius-Schur indicator formula of $V$ introduced in [16]:

$$
\nu_{n}(V)=\chi_{V}\left(\Lambda^{[n]}\right)
$$

where $\chi_{V}$ is the character of $H$ afforded by $V, \Lambda$ is the normalized integral of $H$ and $\Lambda^{[n]}=\Lambda_{1} \Lambda_{2} \cdots \Lambda_{n}$. Here, $x_{1} \otimes \cdots \otimes x_{n}$ denotes the Sweedler notation of the $n$-fold comultiplication of $x \in H$.

The Frobenius-Schur exponent, denoted by $\operatorname{FSexp}(\mathcal{C})$, of a spherical category $\mathcal{C}$ (cf. [26]) is the least positive integer $n$ such that $\nu_{n}(V)=d(V)$ for all $V \in \mathcal{C}$. If such an integer does not exist, $\operatorname{FSexp}(\mathcal{C})$ is defined to be $\infty$. However, the FrobeniusSchur exponent of a spherical fusion category is always finite because the following theorem proved in [26, Thm. 4.1 and 5.5].

Theorem 2.1. Let $\mathcal{C}$ be a spherical fusion category, $\theta$ the twist of $\mathcal{Z}(\mathcal{C})$ and $K$ : $\mathcal{C} \rightarrow \mathcal{Z}(\mathcal{C})$ the two-sided adjoint of the forgetful functor $F: \mathcal{Z}(\mathcal{C}) \rightarrow \mathcal{C}$. Then, for $V \in \mathcal{C}$ 
(i) $\nu_{n}(V)=\frac{1}{\operatorname{dim} \mathcal{C}} \underline{\operatorname{ptr}}\left(\theta_{K(V)}\right)$ for all $V \in \mathcal{C}$, and

(ii) $\operatorname{FSexp}(\mathcal{C})=\operatorname{ord}(\theta)$.

For a simple object $V \in \mathcal{C}$, it is known that $\nu_{1}(V)=1$ if $V \cong \mathbf{1}$, and 0 otherwise. The second indicator of $V$ can only be $0,1,-1$ depending on whether $V$ is selfdual or not. By Theorem 2.1, we know $\nu_{N}(V)=d(V)$ if $N=\operatorname{FSexp}(\mathcal{C})$. The meaning of higher indicators of $V$ are more obscure and they are not rational integers in general (cf. [13, Ex. 7.5]). The theorem implies that the indicator sequence $\nu(V):=\left\{\nu_{n}(V)\right\}_{n \in \mathbb{N}}$ of $V$ is periodic for any object $V$ of a spherical fusion category $\mathcal{C}$. Moreover, $\operatorname{FSexp}(\mathcal{C})$ is the global period of all the indicator sequences of $\mathcal{C}$. The average value or the sum of these indicators over a period should also be an important invariant.

Definition 2.2. Let $\mathcal{C}$ be a spherical fusion category and $N=\mathrm{FSexp}(\mathcal{C})$. The total Frobenius-Schur indicator of $V \in \mathcal{C}$, denoted by $\bar{\nu}(V)$, is defined as

$$
\bar{\nu}(V):=\sum_{i=1}^{N} \nu_{i}(V) .
$$

To prove Theorem A, we first derive a formula for the total indicator $\bar{\nu}(V)$ of an object $V$ in a spherical fusion category $\mathcal{C}$ in terms of some data of the center $\mathcal{Z}(\mathcal{C})$. Recall from [22, Proposition 8.1] that the forgetful functor $F: \mathcal{Z}(\mathcal{C}) \rightarrow \mathcal{C}$ has a two-sided adjoint $K: \mathcal{C} \rightarrow \mathcal{Z}(\mathcal{C})$. It follows from the semisimplicity of $\mathcal{C}$, we have

$$
K(V) \cong \sum_{X \in \operatorname{Irr}(\mathcal{Z}(\mathcal{C}))} \operatorname{dim}(\mathcal{Z}(\mathcal{C})(K(V), X)) X
$$

for $V \in \mathcal{C}$, where $\operatorname{Irr}(\mathcal{Z}(\mathcal{C}))$ is a complete set of non-isomorphic simple objects of $\mathcal{Z}(\mathcal{C})$. For simplicity, we set

$$
[U: V]_{\mathcal{C}}:=\operatorname{dim}(\mathcal{C}(U, V)) \text { for all } U, V \in \mathcal{C} .
$$

Since $K$ is a left adjoint of $F, \mathcal{Z}(\mathcal{C})(K(V), X) \cong \mathcal{C}(V, F(X))$ and so

$$
[K(V): X]_{\mathcal{Z}(\mathcal{C})}=[V: F(X)]_{\mathcal{C}}
$$

for $X \in \mathcal{Z}(\mathcal{C})$ and $V \in \mathcal{C}$.

Since $\operatorname{dim}(\mathcal{C}(U, V))=\operatorname{dim}(\mathcal{C}(V, U))=\operatorname{dim}\left(\mathcal{C}\left(U^{*}, V^{*}\right)\right)$, we have

$$
[V: U]_{\mathcal{C}}=[U: V]_{\mathcal{C}}=\left[U^{*}, V^{*}\right]_{\mathcal{C}} \text { for all } U, V \in \mathcal{C} .
$$

Thus, we have

$$
K(V) \cong \sum_{X \in \operatorname{Irr}(\mathcal{Z}(\mathcal{C}))}[F(X): V]_{\mathcal{C}} X .
$$

Let $\theta$ be the twist of $\mathcal{Z}(\mathcal{C})$. For any $X \in \operatorname{Irr}(\mathcal{Z}(\mathcal{C}))$, we define $\omega_{X} \in \mathbb{C}$ by the equation

$$
\theta_{X}=\omega_{X} \operatorname{id}_{X} .
$$

Note that $\omega_{X}$ is an $N$-th root of unity by Theorem 2.1 .

Proposition 2.3. Let $\mathcal{C}$ be a spherical fusion categories over $\mathbb{C}$ with $\operatorname{FSexp}(\mathcal{C})=N$, and $V \in \mathcal{C}$. Then

(i) $\bar{\nu}(V)$ is an algebraic integer invariant under pivotal equivalence, i.e. if $\mathcal{F}$ : $\mathcal{C} \rightarrow \mathcal{D}$ defines an equivalence of pivotal categories, then $\bar{\nu}(V)=\bar{\nu}(\mathcal{F}(V))$. 
(ii) Moreover,

$$
\bar{\nu}(V)=\frac{N}{\operatorname{dim}(\mathcal{C})} \sum_{\substack{X \in \operatorname{Irr}(\mathcal{Z}(\mathcal{C})) \\ \theta_{X}=\operatorname{id} X}}[F(X): V]_{\mathcal{C}} d(X)
$$

where $F: \mathcal{Z}(\mathcal{C}) \rightarrow \mathcal{C}$ is the forgetful functor.

Proof. Statement (i) is an immediate consequence of the fact that $\nu_{n}(V)$ are algebraic integers for all $n$, and that both $\nu_{n}(V)$ and $\operatorname{FSexp}(\mathcal{C})$ are invariant under pivotal equivalences.

(ii) By Theorem 2.1, we have

$$
\nu_{n}(V)=\frac{1}{\operatorname{dim}(\mathcal{C})} \underline{\operatorname{ptr}}\left(\theta_{K(V)}^{n}\right)=\frac{1}{\operatorname{dim}(\mathcal{C})} \sum_{X \in \operatorname{Irr}(\mathcal{Z}(\mathcal{C}))}[F(X): V]_{\mathcal{C}} \omega_{X}^{n} d(X) .
$$

Therefore,

$$
\begin{aligned}
\bar{\nu}(V) & =\sum_{i=1}^{N} \nu_{i}(V) \\
& =\frac{1}{\operatorname{dim}(\mathcal{C})} \sum_{i=1}^{N} \sum_{X \in \operatorname{Irr}(\mathcal{Z}(\mathcal{C}))}[F(X): V]_{\mathcal{C}} \omega_{X}^{i} d(X) \\
& =\frac{1}{\operatorname{dim}(\mathcal{C})} \sum_{X \in \operatorname{Irr}(\mathcal{Z}(\mathcal{C}))}[F(X): V]_{\mathcal{C}} \sum_{i=1}^{N} \omega_{X}^{i} d(X) \\
& =\frac{N}{\operatorname{dim}(\mathcal{C})} \sum_{\substack{X \in \operatorname{Irr}(\mathcal{Z}(\mathcal{C})) \\
\omega_{X}=1}}[F(X): V]_{\mathcal{C}} d(X) .
\end{aligned}
$$

Here, the last equality follows from the fact that $\omega_{X}$ is an $N$-th root of unity.

We can now prove Theorem A.

Proof of Theorem A. Let $H$ be a semisimple quasi-Hopf algebra. Consider the canonical pivotal structure on $\operatorname{Rep}(H)$. Then $\operatorname{Rep}(H)$ is a spherical fusion category with $d(V)=\operatorname{dim} V$ for all $V \in \operatorname{Rep}(H)$. In particular, $\operatorname{dim}(\operatorname{Rep}(H))=\operatorname{dim} H$ and $d(X)$ is a non-negative integer for all $X \in \mathcal{Z}(\operatorname{Rep}(H))$. It follows from Proposition 2.3 (ii) that

$$
\bar{\nu}(V)=\frac{N}{\operatorname{dim} H} \sum_{\substack{X \in \operatorname{Irr}(\mathcal{Z}(\mathcal{C})) \\ \omega_{X}=1}}[F(X): V]_{\mathcal{C}} d(X)
$$

is a non-negative rational number. By Proposition $2.3(\mathrm{i}), \bar{\nu}(V)$ is a non-negative integer. Since

$$
\frac{\bar{\nu}(V) \operatorname{dim} H}{N}=\sum_{\substack{X \in \operatorname{Irr}(\mathcal{Z}(\mathcal{C})) \\ \omega_{X}=1}}[F(X): V]_{\mathcal{C}} d(X) \in \mathbb{Z},
$$

we establish the divisibility $N \mid(\operatorname{dim} H) \bar{\nu}(V)$.

If $H$ is a semisimple Hopf algebra, $\exp (H)=\operatorname{ord}(\theta)=\mathrm{FSexp}(H)$ (cf. [6, Thm 2.5] and Theorem 2.1). Therefore, Theorem A is related to the following well-known conjecture considered by Kashina [11, 12]. 
Conjecture 2.4. Let $H$ be a semisimple Hopf algebra over $\mathbb{C}$. Then the exponent of $H$ divides $\operatorname{dim}(H)$.

By the Cauchy theorem for Hopf algebras [13, Sect. 6] (see so [26, Thm. 8.4]), $\operatorname{dim} H$ and $\exp (H)$ have the same prime factors. Thus, if $\operatorname{gcd}\{\bar{\nu}(V) \mid V \in \operatorname{Irr}(H)\}$ is relatively prime to $\operatorname{dim} H$, then the conjecture will be proved for $H$. However, the Kac algebra $K$ of dimension 8 is an example in which $\operatorname{gcd}\{\bar{\nu}(V) \mid V \in \operatorname{Irr}(K)\}=4$ (see Example 3.6). Nevertheless, any upper bound of $\operatorname{gcd}\{\bar{\nu}(V) \mid V \in \operatorname{Irr}(H)\}$ will shed light on the conjecture.

By Theorem A, $\bar{\nu}(V)=0$ is an extreme value and it is possible for some quasiHopf algebra demonstrated in the following example.

Example 2.5. Let $G$ be a finite group and $\omega$ a normalized 3-cocycle on $G$ with coefficients in $\mathbb{C}^{\times}$. Following [25, Sect. 7], one can construct a quasi-Hopf algebra $H(G, \omega)=\left(\mathbb{C}[G]^{*}, \Delta, \varepsilon, \phi, \alpha, \beta, S\right)$ where multiplication, identity, comultiplication $\Delta$, counit $\varepsilon$, and antipode $S$ are the same as the structure maps of the dual $\mathbb{C}[G]^{*}$ of the group algebra $\mathbb{C}[G]$, and $\phi, \alpha$, and $\beta$ are given by

$\phi=\sum_{a, b, c \in G} \omega(a, b, c) e(a) \otimes e(b) \otimes e(c), \quad \alpha=1, \quad$ and $\quad \beta=\sum_{a \in G} \omega\left(a, a^{-1}, a\right)^{-1} e(a)$,

where $\{e(x) \mid x \in G\}$ is the dual basis of $G$ for $\mathbb{C}[G]^{*}$. If $\omega^{\prime} \in Z^{3}\left(G, \mathbb{C}^{\times}\right)$is cohomologous to $\omega$, then $H(G, \omega)$ can be twisted to the quasi-bialgebra $H\left(G, \omega^{\prime}\right)$ by a gauge transformation (see $[14, \mathrm{XV}]$ for gauge equivalence of quasi-bialgebras). In particular, if $\omega$ is a coboundary of the 2-cochain $f: G \times G \rightarrow \mathbb{C}^{\times}$, then $H(G, \omega)$ can be twisted by the gauge transformation $F=\sum_{a, b \in G} f(a, b) e(a) \otimes e(b)$ to the ordinary bialgebra $\mathbb{C}[G]^{*}$.

We now consider a special case when $G$ is of order 2 . Let $G=\{1, x\}$ be an abelian group of order 2 and $\omega$ a 3-cocycle of $G$ given by

$$
\omega(a, b, c)=\left\{\begin{aligned}
-1 & \text { if } a=b=c=x, \\
1 & \text { otherwise. }
\end{aligned}\right.
$$

Then $H=H(G, \omega)$ is a 2-dimensional commutative quasi-Hopf algebra. Let $V$ be the nontrivial simple $H$-module. As computed in [26, Ex. 5.4], $\operatorname{FSexp}(H)=4$ and $\nu_{n}(V)=\cos \left(\frac{n \pi}{2}\right)$. Therefore, $\bar{\nu}(V)=0$.

\section{Proof of Theorem B}

Example 2.5 shows that $\bar{\nu}(V)=0$ for some simple module $V$ of a semisimple quasi-Hopf algebra. However, this cannot happen for semisimple Hopf algebras which is stated in Theorem B. We will prove this theorem in this section.

Recall that the Drinfeld double $D(H)$ of a finite-dimensional Hopf algebra $H$ is the bicrossproduct $H^{* \text { cop }} \bowtie H$ (cf. $[19,14]$ ). In particular, $D(H)$ is a finitedimensional Hopf algebra with comultiplication and multiplication of $D(H)$ described by

$$
\Delta(f \bowtie a)=\left(f_{2} \bowtie a_{1}\right) \otimes\left(f_{1} \bowtie a_{2}\right)
$$

and

$$
(f \bowtie a)(g \bowtie b)=f\left(g\left(S^{-1}\left(a_{3}\right) ? a_{1}\right)\right) \bowtie a_{2} b
$$

respectively, for $f, g \in H^{*}$ and $a, b \in H$. Here $a_{1} \otimes a_{2}$ and $f_{1} \otimes f_{2}$ are respectively the Sweedler notation for the comultiplications of $a \in H$ and $f \in H^{*}$ with the 
summation suppressed. The Hopf algebra $D(H)$ admits a canonical universal $\mathcal{R}$ matrix $R=\sum_{i} \varepsilon \bowtie h_{i} \otimes h^{i} \bowtie 1$ where $\left\{h_{i}\right\}_{i}$ and $\left\{h^{i}\right\}_{i}$ are dual bases of $H$ and $H^{*}$ respectively.

It should be well-known to experts that $\operatorname{Rep}(D(H))$ and $\operatorname{Rep}\left(D\left(H^{*}\right)\right)$ are equivalent braided monoidal categories. For the sake of completeness, we provide a proof of the statement in terms of gauge equivalence for subsequent discussion.

Lemma 3.1. Let $H$ be a finite-dimensional Hopf algebra over any field $\mathbb{k}$, not necessarily semisimple. Then $D(H)$ and $D\left(H^{*}\right)$ are gauge equivalent quasi-triangular Hopf algebras via the gauge transformation $R \in D\left(H^{*}\right) \otimes D\left(H^{*}\right)$, which is the universal $\mathcal{R}$-matrix of $D\left(H^{*}\right)$, and the algebra isomorphism

$$
\sigma: D(H) \rightarrow D\left(H^{*}\right), \quad f \bowtie a \mapsto(1 \bowtie f)(a \bowtie \varepsilon) .
$$

Here, $H^{* *}$ is identified with $H$ as Hopf algebras under the natural isomorphism $j: H \rightarrow H^{* *}$ of vector spaces.

Proof. Since $\Delta_{D\left(H^{*}\right)}^{\mathrm{op}}(x)=R \Delta_{D\left(H^{*}\right)}(x) R^{-1}$ for all $x \in D\left(H^{*}\right)$, it suffices to show that $\sigma: D(H) \rightarrow D\left(H^{*}\right)^{\text {cop }}$ is a bialgebra homomorphism, but this is straightforward verification.

The algebra isomorphism $\sigma: D(H) \rightarrow D\left(H^{*}\right)$ defines a braided monoidal equivalence $\left(\mathcal{F}_{\sigma}, \xi\right.$, id $): \operatorname{Rep}\left(D\left(H^{*}\right)\right) \rightarrow \operatorname{Rep}(D(H))$ (cf. [14, XIII.3.2]). Here, $\mathcal{F}_{\sigma}: \operatorname{Rep}\left(D\left(H^{*}\right)\right) \rightarrow \operatorname{Rep}(D(H))$ is the $\mathbb{C}$-linear functor with $\mathcal{F}_{\sigma}(V)$ defined as the $D(H)$-module via the isomorphism $\sigma$ for $V \in \operatorname{Rep}\left(D\left(H^{*}\right)\right)$, and $\mathcal{F}_{\sigma}$ : $\operatorname{Hom}_{D\left(H^{*}\right)}(V, W) \rightarrow \operatorname{Hom}_{D(H)}\left(\mathcal{F}_{\sigma} V, \mathcal{F}_{\sigma} W\right)$ the identity function. The coherence isomorphism $\xi: \mathcal{F}_{\sigma}(V) \otimes \mathcal{F}_{\sigma}(W) \rightarrow \mathcal{F}_{\sigma}(V \otimes W)$ is the left action of $R^{-1}$, and $\mathcal{F}_{\sigma}\left(\mathbf{1}_{D\left(H^{*}\right)}\right)=\mathbf{1}_{D(H)}$.

The Hopf algebra $H^{*}$ can be considered as a subalgebra of $D(H)$ and $D\left(H^{*}\right)$ via the embeddings $i_{1}: H^{*} \rightarrow D\left(H^{*}\right)$ and $i_{2}: H^{*} \rightarrow D(H)$ defined by

$$
i_{1}(f)=1 \bowtie f, \quad i_{2}(f)=f \bowtie 1
$$

respectively, for $f \in H^{*}$. It follows immediately from the definition of $\sigma$ that we have the commutative diagram of algebra maps:

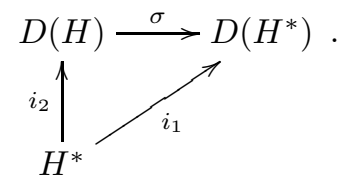

This implies the following lemma for the two pairs of induction and restriction functors.

Lemma 3.2. Let $\operatorname{Res}_{H^{*}}^{D\left(H^{*}\right)}$ and $\operatorname{Res}_{H^{*}}^{D(H)}$ be the restriction functors along the embeddings $i_{1}$ and $i_{2}$ respectively, and $\operatorname{Ind}_{H^{*}}^{D\left(H^{*}\right)}$ and $\operatorname{Ind}_{H^{*}}^{D(H)}$ the associated induction functors. Then we have

$$
\mathcal{F}_{\sigma} \circ \operatorname{Ind}_{H^{*}}^{D\left(H^{*}\right)}=\operatorname{Ind}_{H^{*}}^{D(H)} \quad \text { and } \quad \operatorname{Res}_{H^{*}}^{D(H)} \circ \mathcal{F}_{\sigma}=\operatorname{Res}_{H^{*}}^{D\left(H^{*}\right)} .
$$

The preceding lemmas hold for any finite-dimensional Hopf algebras. We now turn to semisimple Hopf algebras $H$. In this case, $\mathcal{C}=\operatorname{Rep}(H)$ is a spherical fusion category, and the right center $\mathcal{Z}(\mathcal{C})$ of $\mathcal{C}$ is equivalent to the $\operatorname{Rep}(D(H))$ as braided monoidal category. Equipped with the canonical pivotal structure, $\operatorname{Rep}(D(H))$ is a modular tensor category with the twist $\theta$ given by the action of the Drinfeld 
element of $D(H)$. The forgetful functor $F: \mathcal{Z}(\mathcal{C}) \rightarrow \mathcal{C}$ is the restriction $\operatorname{Res}_{H}^{D(H)}$ which has a left adjoint $\operatorname{Ind}_{H}^{D(H)}: \mathcal{C} \rightarrow \mathcal{Z}(\mathcal{C})$. Thus, by the uniqueness of adjoint functor, $K: \mathcal{Z}(\mathcal{C}) \rightarrow \mathcal{C}$ is equivalent to $\operatorname{Ind}_{H}^{D(H)}$. If $V$ is a self-dual $H$-module, then so is $K(V)$. This observation even holds for spherical fusion categories.

Lemma 3.3. Let $\mathcal{C}$ be a spherical fusion category, $\mathcal{Z}(\mathcal{C})$ the center of $\mathcal{C}$ and $K$ : $\mathcal{C} \rightarrow \mathcal{Z}(\mathcal{C})$ the left adjoint of the forgetful functor $F: \mathcal{Z}(\mathcal{C}) \rightarrow \mathcal{C}$. Then $K(V)$ is a self-dual object of $\mathcal{Z}(\mathcal{C})$ whenever $V \in \mathcal{C}$ is self-dual. In particular, $K\left(\mathbf{1}_{\mathcal{C}}\right)$ is self-dual. Moreover, if $\theta$ is the twist of $\mathcal{Z}(\mathcal{C})$, then $\theta_{K\left(\mathbf{1}_{\mathcal{C}}\right)}=\operatorname{id}_{K\left(\mathbf{1}_{\mathcal{C}}\right)}$.

Proof. Note that for $X \in \operatorname{Irr}(\mathcal{Z}(\mathcal{C})), X^{*}$ is isomorphic to a unique object of $\operatorname{Irr}(\mathcal{Z}(\mathcal{C}))$. Let $V \in \mathcal{C}$ be self-dual. It follows from $(2.2)$ that

$$
\begin{aligned}
K(V)^{*} & \cong \sum_{X \in \operatorname{Irr}(\mathcal{Z}(\mathcal{C}))}[F(X): V]_{\mathcal{C}} X^{*} \\
& \cong \sum_{X \in \operatorname{Irr}(\mathcal{Z}(\mathcal{C}))}\left[F\left(X^{*}\right): V\right]_{\mathcal{C}} X \\
& \cong \sum_{X \in \operatorname{Irr}(\mathcal{Z}(\mathcal{C}))}\left[F(X)^{*}: V\right]_{\mathcal{C}} X \\
& \cong \sum_{X \in \operatorname{Irr}(\mathcal{Z}(\mathcal{C}))}\left[F(X)^{*}: V^{*}\right]_{\mathcal{C}} X \\
& \cong \sum_{X \in \operatorname{Irr}(\mathcal{Z}(\mathcal{C}))}[F(X): V]_{\mathcal{C}} X \\
& \cong K(V) .
\end{aligned}
$$

Here, the third isomorphism is a consequence of the fact that the forgetful functor $F: \mathcal{Z}(\mathcal{C}) \rightarrow \mathcal{C}$ defines a monoidal functor, and the last isomorphism follows from the remark preceding Proposition 2.3. Since $\mathbf{1}_{\mathcal{C}}$ is self-dual, and so is $K\left(\mathbf{1}_{\mathcal{C}}\right)$.

By [28, Prop. 2.8 (iii)], $\left[F(X): \mathbf{1}_{\mathcal{C}}\right]_{\mathcal{C}} \neq 0$ implies $\omega_{X}=1$. Therefore,

$$
\theta_{K\left(\mathbf{1}_{\mathcal{C}}\right)}=\sum_{X \in \operatorname{Irr}(\mathcal{Z}(\mathcal{C}))}\left[F(X): \mathbf{1}_{\mathcal{C}}\right]_{\mathcal{C}} \theta_{X}=\sum_{X \in \operatorname{Irr}(\mathcal{Z}(\mathcal{C}))}\left[F(X): \mathbf{1}_{\mathcal{C}}\right]_{\mathcal{C}} \operatorname{id}_{X}=\operatorname{id}_{K\left(\mathbf{1}_{\mathcal{C}}\right)} .
$$

This proves the last assertion.

We can now prove Theorem B.

Proof of Theorem B. Let $\mathcal{C}=\operatorname{Rep}(H)$. In view of Proposition 2.3 (ii), it suffices to show the inequality

$$
\sum_{\substack{X \in \operatorname{Irr}(D(H)) \\ \theta_{X}=\operatorname{id} X}}\left[\operatorname{Res}_{H}^{D(H)} X: V\right]_{\mathcal{C}} \operatorname{dim}(X) \geq \operatorname{dim}(V)
$$

for all $V \in \mathcal{C}$. This is equivalent to show that $\left[\operatorname{Res}_{H}^{D(H)} W: V\right]_{\mathcal{C}}>0$ for some $D(H)$-module $W$ satisfying $\theta_{W}=\mathrm{id}_{W}$.

Recall that $H^{*}$ is also a semisimple Hopf algebra [15]. By Lemma 3.3, $Y=$ $\operatorname{Ind}_{H^{*}}^{D\left(H^{*}\right)}\left(\mathbf{1}_{H^{*}}\right)$ is a self-dual $D\left(H^{*}\right)$-module and $\theta_{Y}=\mathrm{id}_{Y}$. Moreover,

$$
Y \cong \sum_{U \in \operatorname{Irr}\left(D\left(H^{*}\right)\right)}\left[U: \mathbf{1}_{H^{*}}\right]_{\mathcal{C}^{\prime}} U
$$


where $\mathcal{C}^{\prime}=\operatorname{Rep}\left(H^{*}\right)$. Since $\operatorname{Rep}\left(D\left(H^{*}\right)\right)$ and $\operatorname{Rep}(D(H))$ are spherical categories equipped with their canonical pivotal structures, $\mathcal{F}_{\sigma}: \operatorname{Rep}\left(D\left(H^{*}\right)\right) \rightarrow \operatorname{Rep}(D(H))$ defines an equivalence of braided monoidal categories as well as pivotal categories. Therefore, by [9, Prop. 6.2], we have

$$
\operatorname{id}_{\mathcal{F}_{\sigma} Y}=\mathcal{F}_{\sigma}\left(\operatorname{id}_{Y}\right)=\mathcal{F}_{\sigma}\left(\theta_{Y}\right)=\theta_{\mathcal{F}_{\sigma} Y} .
$$

In particular, $W=\mathcal{F}_{\sigma} Y$ is a self-dual $D(H)$-module satisfying $\theta_{W}=\mathrm{id}_{W}$. Since $\operatorname{Ind}_{H}^{D(H)}$ is a left adjoint of $\operatorname{Res}_{H}^{D(H)}$, we have

$$
\left[V: \operatorname{Res}_{H}^{D(H)} W\right]_{\mathcal{C}}=\left[\operatorname{Ind}_{H}^{D(H)} V: W\right]_{\mathcal{Z}(\mathcal{C})} .
$$

However, by Lemma 3.2, $W=\operatorname{Ind}_{H^{*}}^{D(H)} \mathbf{1}_{H^{*}}$. Therefore,

$$
\begin{aligned}
\operatorname{Hom}_{D(H)}\left(\operatorname{Ind}_{H}^{D(H)} V, W\right) & =\operatorname{Hom}_{D(H)}\left(\operatorname{Ind}_{H}^{D(H)} V, \operatorname{Ind}_{H^{*}}^{D(H)} \mathbf{1}_{H^{*}}\right) \\
& \cong \operatorname{Hom}_{D(H)}\left(\left(\operatorname{Ind}_{H^{*}}^{D(H)} \mathbf{1}_{H^{*}}\right)^{*} \otimes\left(\operatorname{Ind}_{H}^{D(H)} V\right), \mathbf{1}_{D(H)}\right) \\
& \cong \operatorname{Hom}_{D(H)}\left(\left(\operatorname{Ind}_{H^{*}}^{D(H)} \mathbf{1}_{H^{*}}\right) \otimes\left(\operatorname{Ind}_{H}^{D(H)} V\right), \mathbf{1}_{D(H)}\right) .
\end{aligned}
$$

By $[4$, Thm. 8 (1)], we have

$$
\left(\operatorname{Ind}_{H^{*}}^{D(H)} \mathbf{1}_{H^{*}}\right) \otimes\left(\operatorname{Ind}_{H}^{D(H)} V\right) \cong D(H)^{\operatorname{dim} V}
$$

as $D(H)$-modules. Thus,

$$
\left[\operatorname{Ind}_{H}^{D(H)} V: W\right]_{\mathcal{Z}(\mathcal{C})}=\left[D(H)^{\operatorname{dim} V}: \mathbf{1}_{D(H)}\right]_{\mathcal{Z}(\mathcal{C})}=\operatorname{dim} V,
$$

and so $\left[V: \operatorname{Res}_{H}^{D(H)} W\right]_{\mathcal{C}}=\operatorname{dim} V$. This completes the proof.

Definition 3.4. A quasi-Hopf algebra $H$ is said to be genuine if $H$ is not gauge equivalent to any ordinary Hopf algebra.

Theorem B provides a sufficient condition for a semisimple quasi-Hopf algebra being genuine.

Corollary 3.5. Let $H$ be a semisimple quasi-Hopf algebra. If there exists $V \in$ $\operatorname{Rep}(H)$ such that $\bar{\nu}(V)=0$, then $H$ is a genuine quasi-Hopf algebra.

Proof. Assume contrary. Then $H$ is gauge equivalent to a Hopf algebra $H^{\prime}$. There exists a monoidal equivalence $\mathcal{F}: \operatorname{Rep}(H) \rightarrow \operatorname{Rep}\left(H^{\prime}\right)$ (see [25, Thm. 2.2]). Thus, $\mathcal{F}$ preserves their canonical pivotal structures (cf. [27]). By Proposition 2.3 (i) and Theorem B, we have

$$
\bar{\nu}(V)=\bar{\nu}(\mathcal{F}(V))>0
$$

for all $V \in \operatorname{Rep}(H)$.

The following example shows that the existence of vanishing total indicators can also be a necessary condition for a genuine quasi-Hopf algebra.

Example 3.6. There are exactly four gauge inequivalent 8-dimensional quasi-Hopf algebras with five simple objects $\left\{a_{1}, a_{2}, a_{3}, a_{4}, m\right\}$ and fusion rules given by

$$
\left\{a_{1}, a_{2}, a_{3}, a_{4}\right\} \cong \mathbb{Z}_{2} \times \mathbb{Z}_{2}, \quad m^{2} \cong \sum_{i=1}^{4} a_{i} \text { and } m a_{i} \cong a_{i} m \cong m
$$

for $i=1,2,3,4$. Their categories of representations are Tambara-Yamagami categories $[31]$. They are $\mathbb{C}[Q], \mathbb{C}[D]$, the 8-dimensional Kac algebra $K$ and its twisted 
version $K_{u}$ (see [25, Sec.6] for details), where $Q$ and $D$ are, respectively, the quaternion group and the dihedral group of order 8 . We can assume $a_{1}$ to be the unit object. Then $\nu_{n}\left(a_{1}\right)=1$ for all $n \geq 1$. The objects $a_{2}, a_{3}$ and $a_{4}$ are 1-dimensional representations of these algebras, and their orders are 2 . The indicator $\nu_{n}\left(a_{i}\right)=1$ if $n$ is even, and 0 otherwise, for $i=2,3,4$. Thus $\bar{\nu}\left(a_{i}\right)>0$ for $i=1,2,3,4$. By [25, Sec. 6], we find the following table:

\begin{tabular}{|c|c|c|c|c|c|c|c|c|}
\hline & $\nu_{2}(m)$ & $\nu_{3}(m)$ & $\nu_{4}(m)$ & $\nu_{5}(m)$ & $\nu_{6}(m)$ & $\nu_{7}(m)$ & $\nu_{8}(m)$ & $\bar{\nu}(m)$ \\
\hline$K$ & 1 & 0 & 0 & 0 & 1 & 0 & 2 & 4 \\
\hline$K_{u}$ & -1 & 0 & 0 & 0 & -1 & 0 & 2 & 0 \\
\hline $\mathbb{C}[D]$ & 1 & 0 & 2 & 0 & 1 & 0 & 2 & 3 \\
\hline $\mathbb{C}[Q]$ & -1 & 0 & 2 & 0 & -1 & 0 & 2 & 1 \\
\hline
\end{tabular}

In particular, the Frobenius-Schur exponents of $K, K_{u}, \mathbb{C}[D], \mathbb{C}[Q]$ are 8, 8, 4, 4 respectively. The quasi-Hopf algebra $K_{u}$ is the only one in the list for which $\bar{\nu}(m)=0$. Therefore, $K_{u}$ is a genuine quasi-Hopf algebra. It is well-known that $K, \mathbb{C}[D], \mathbb{C}[Q]$ are all the noncommutative semisimple Hopf algebras of dimension 8 (cf. [18]). Their corresponding indicators $\nu_{n}(m)$ can be computed using the formula $(2.1)$.

\section{Twisted QuAntum DOUbles}

The main result of this section is to show that existence of vanishing total indicators is also a necessary condition for an abelian twisted quantum double, considered in [23], being genuine.

The twisted quantum double $D^{\omega}(G)$ of $G$ relative to a normalized 3-cocycle $\omega$ : $G \times G \times G \rightarrow \mathbb{C}^{\times}$is the semisimple quasi-Hopf algebra with underlying vector space $\mathbb{C}[G]^{*} \otimes \mathbb{C}[G]$ in which multiplication, comultiplication $\Delta$, associator $\phi$, counit $\varepsilon$ and quasi-antipode $(S, \alpha, \beta)$ are given by

$$
\begin{aligned}
& (e(g) \otimes x)(e(h) \otimes y)=\theta_{g}(x, y) \delta_{g^{x}, h} e(g) \otimes x y, \\
& \Delta(e(g) \otimes x)=\sum_{h k=g} \gamma_{x}(h, k) e(h) \otimes x \otimes e(k) \otimes x, \\
& \phi=\sum_{g, h, k \in G} \omega(g, h, k)^{-1} e(g) \otimes 1 \otimes e(h) \otimes 1 \otimes e(k) \otimes 1, \\
& \varepsilon(e(g) \otimes x)=\delta_{g, 1}, \quad \alpha=1, \quad \beta=\sum_{g \in G} \omega\left(g, g^{-1}, g\right) e(g) \otimes 1, \\
& S(e(g) \otimes x)=\theta_{g^{-1}}\left(x, x^{-1}\right)^{-1} \gamma_{x}\left(g, g^{-1}\right)^{-1} e\left(x^{-1} g^{-1} x\right) \otimes x^{-1},
\end{aligned}
$$

where $\{e(g) \mid g \in G\}$ is the dual basis of $\{g \mid g \in G\}, \delta_{g, 1}$ is the Kronecker delta, $g^{x}=x^{-1} g x$, and

$$
\begin{aligned}
& \theta_{g}(x, y)=\frac{\omega(g, x, y) \omega\left(x, y,(x y)^{-1} g x y\right)}{\omega\left(x, x^{-1} g x, y\right)} \\
& \gamma_{g}(x, y)=\frac{\omega(x, y, g) \omega\left(g, g^{-1} x g, g^{-1} y g\right)}{\omega\left(x, g, g^{-1} y g\right)}
\end{aligned}
$$

for any $x, y, g \in G(\mathrm{cf} .[5,14])$.

As in [23], we denote by $\Gamma^{\omega}$ the set of all group-like elements of $D^{\omega}(G)$ and call $\omega$ abelian if $D^{\omega}(G)$ is a commutative algebra. This can only happen when $G$ is abelian and $\Gamma^{\omega}$ spans $D^{\omega}(G)$. The following theorem characterizes those abelian twisted 
doubles of finite groups which are genuine quasi-Hopf algebras, and it provides an answer for a question of Dijkgraaf, Pasquier and Roche posed in [5, p69].

Theorem 4.1. Let $G$ be a finite abelian group, and $\omega$ a normalized 3-cocycle of $G$ such that $D^{\omega}(G)$ is a commutative algebra. Then $D^{\omega}(G)$ is a genuine quasi-Hopf algebra if, and only if, there exists $V \in \operatorname{Rep}\left(D^{\omega}(G)\right)$ such that $\bar{\nu}(V)=0$.

To prove this theorem, we need some preparations. Recall that an EilenbergMacLane (EM) 3-cocycle of $G$ is a pair $(\omega, c)$ where $\omega \in Z^{3}\left(G, \mathbb{C}^{\times}\right)$and $c$ a 2-cochain of $G$ satisfying the following conditions:

$$
\frac{c(x y, z)}{c(x, z) c(y, z)} \frac{\omega(x, z, y)}{\omega(x, y, z) \omega(z, x, y)}=1=\frac{c(x, y z)}{c(x, y) c(x, z)} \frac{\omega(x, y, z) \omega(y, z, x)}{\omega(y, x, z)}
$$

for all $x, y, z \in G$. The EM 3-cocycle $(\omega, c)$ is a coboundary if there exists a 2cochain $h$ of $G$ such that

$$
\omega=\delta h \quad \text { and } \quad c(x, y)=\frac{h(x, y)}{h(y, x)} .
$$

The EM cohomology group $\mathrm{H}_{a b}^{3}\left(G, \mathbb{C}^{\times}\right)$is then defined by

$$
\mathrm{H}_{a b}^{3}\left(G, \mathbb{C}^{\times}\right)=\frac{Z_{a b}^{3}\left(G, \mathbb{C}^{\times}\right)}{B_{a b}^{3}\left(G, \mathbb{C}^{\times}\right)}
$$

where $Z_{a b}^{3}\left(G, \mathbb{C}^{\times}\right)$and $B_{a b}^{3}\left(G, \mathbb{C}^{\times}\right)$are respectively the abelian groups of EM 3cocycles and 3-coboundaries.

For $(\omega, c) \in Z_{a b}^{3}\left(G, \mathbb{C}^{\times}\right)$, one can assign the function $t(x):=c(x, x)$, called its trace. The trace $t$ of an EM 3-cocycle of $G$ is a quadratic function on $G$, that means

(i) $t\left(x^{a}\right)=t(x)^{a^{2}}$ for $a \in \mathbb{Z}$, and

(ii) $b_{t}(x, y):=\frac{t(x y)}{t(x) t(y)}$ defines a bicharacter of $G$.

The following theorem of Eilenberg and Mac Lane is essential to the discussion in this section (cf. [17, Thm. 3] and [10, $§ 7]$ ).

Theorem 4.2 (Eilenberg-MacLane). Let $Q\left(G, \mathbb{C}^{\times}\right)$be the abelian group of all quadratic functions from $G$ to $\mathbb{C}^{\times}$. The map assigning to each 3-cocycle its trace induces an isomorphism

$$
\mathrm{H}_{a b}^{3}\left(G, \mathbb{C}^{\times}\right) \stackrel{\cong}{\rightarrow} Q\left(G, \mathbb{C}^{\times}\right)
$$

Remark 4.3. Recall the example of quasi-Hopf algebra $H(G, \omega)$ defined in Example 2.5 for a normalized 3-cocycle $\omega$ on a finite group $G$. Let $\mathcal{C}=\operatorname{Rep}(H(G, \omega))$. Then $\operatorname{Irr}(\mathcal{C})$ consists of a set of 1-dimensional representations $V_{x}$ indexed by $G$. Moreover, a pair $(\omega, c)$ is an EM 3-cocycle of a finite abelian group $G$ if, and only if, $\operatorname{Rep}(H(G, \omega))$ is a braided spherical fusion category with the braiding given by

$$
c_{V_{x}, V_{y}}:=\left(V_{x} \otimes V_{y} \stackrel{c(x, y)}{\longrightarrow} V_{y} \otimes V_{x}\right)
$$

for $x, y \in G$.

For simplicity, we say that a quadratic function $t: G \rightarrow \mathbb{C}^{\times}$of a finite abelian group $G$ can be obtained from a bicharacter $b$ of $G$ if $t(x)=b(x, x)$ for all $x \in G$. We denote by $K\left(G, \mathbb{C}^{\times}\right)$the set of all quadratic functions which can be obtained from some bicharacters of $G$. 
Lemma 4.4. Let $G$ be a finite abelian group and $(\omega, c)$ an EM 3-cocycle of $G$ with trace $t$. Then:

(i) $\omega$ is a coboundary if, and only if, $t \in K\left(G, \mathbb{C}^{\times}\right)$.

(ii) $\omega^{2}$ is a 3-coboundary of $G$.

Proof. Let us denote the cohomology class represented by the EM 3-cocycle $(\omega, c)$ of $G$ as $[(\omega, c)]$. Then $\omega$ is a coboundary if, and only if, $[(\omega, c)]=[(1, b)]$ for some 2 -cochain $b$ of $G$. By (4.1), $b$ is a bicharacter of $G$ and $t(x)=c(x, x)=b(x, x)$ for all $x \in G$. Therefore, $t \in K\left(G, \mathbb{C}^{\times}\right)$. Conversely, suppose there exists a bicharacter $b$ on $G$ such that $b(x, x)=t(x)$ for all $x \in G$. Since $(1, b)$ is an EM 3-cocycle which has the same trace as $(\omega, c),[(\omega, c)]=[(1, b)]$ by Theorem 4.2. In particular, $\omega$ is a coboundary. This proves statement (i).

(ii) By $(4.1), b(x, y):=c(x, y) c(y, x)$ defines a bicharacter of $G$, and the trace of the EM 3-cocycle $\left(\omega^{2}, c^{2}\right)$ can be obtained from $b$. It follows from (i) that $\omega^{2}$ is a coboundary.

Lemma 4.5. Let $G$ be a finite abelian group and $(\omega, c)$ an EM 3-cocycle of $G$. Then $\omega$ is a coboundary if, and only if, $\bar{\nu}(V)>0$ for all simple $H(G, \omega)$-module $V$. In this case, $H(G, \omega)$ is gauge equivalent to the Hopf algebra $\mathbb{C}[G]^{*}$.

Proof. In view of Example 2.5, if $\omega$ is a coboundary, then $H(G, \omega)$ can be twisted to the bialgebra $\mathbb{C}[G]^{*}$ by a gauge transformation. It follows from Corollary 3.5 that $\bar{\nu}(V)>0$ for all simple $H(G, \omega)$-modules $V$. Conversely, we assume the positivity of total indicators. Let $t$ be the trace of the EM 3-cocycle $(\omega, c)$. By Lemma 4.4(i), it is equivalent to show that $t \in K\left(G, \mathbb{C}^{\times}\right)$. Since $G$ is a direct sum of its cyclic subgroups, by [23, Lem. 6.2(i)], $t \in K\left(G, \mathbb{C}^{\times}\right)$if, and only if, $t_{C} \in K\left(C, \mathbb{C}^{\times}\right)$for each cyclic summand $C$ of $G$, where $t_{C}$ denotes the restriction of $t$ on $C$. Note that the restriction $\left(\omega_{C}, c_{C}\right)$ of $(\omega, c)$ on $C$ is an EM 3-cocycle of $C$ and its trace is equal to $t_{C}$. Therefore, by Lemma $4.4(\mathrm{i})$, it is enough to prove that $\omega_{C}$ is a coboundary for each cyclic subgroup $C$ of $G$.

By Lemma 4.4(ii), $\omega^{2}$ is a coboundary. We may simply assume $\omega^{2}=1$ as $\bar{\nu}(V)$ are preserved by gauge equivalence of semisimple quasi-Hopf algebras. A complete set of non-isomorphic simple $H(G, \omega)$-modules is given by $\operatorname{Irr}(H(G, \omega))=\left\{V_{x} \mid x \in G\right\}$. By [25, Prop. 7.1],

$$
\nu_{n}\left(V_{x}\right)=\delta_{x^{n}, 1} \prod_{r=1}^{n-1} \omega\left(x, x^{r}, x\right) .
$$

The equation implies that $\nu_{n}\left(V_{x}\right)=0$ if $\ell \nmid n$ where $\ell=\operatorname{ord}(x)$. Since $\omega^{2}=1$, $\omega\left(x, x^{r}, x\right)= \pm 1$ for all $r \in \mathbb{Z}$. By $(4.2), \nu_{\ell}\left(V_{x}\right)= \pm 1$ and

$$
\nu_{k \ell}\left(V_{x}\right)=\nu_{\ell}\left(V_{x}\right)^{k} \text {. }
$$

for all positive integer $k$. We claim that $\nu_{\ell}\left(V_{x}\right)=1$. Suppose not. Then $\nu_{\ell}\left(V_{x}\right)=$ -1 . Let $N=\operatorname{FSexp}(H(G, \omega))$. Then $\nu_{N}\left(V_{x}\right)=\operatorname{dim} V_{x}=1$. Therefore, $N / \ell$ is even and so $\bar{\nu}\left(V_{x}\right)=0$ which contradicts the assumption of the positivity of total indicators.

We now have

$$
1=\nu_{\ell}\left(V_{x}\right)=\prod_{r=1}^{\ell-1} \omega\left(x, x^{r}, x\right) .
$$


Note that right hand side is also the $\ell$-th indicator of $V_{x}$ considered as an $H\left(C, \omega_{C}\right)$ module where $C$ is the cyclic subgroup generated by $x$. Since $\nu_{\ell}\left(V_{x}\right)$ is invariant under gauge transformations, the product depends only on the cohomology class of $\omega_{C}$ in $H^{3}\left(C, \mathbb{C}^{\times}\right)$, which is a cyclic group of order $\ell$. A generating 3 -cocycle $\phi$, as described in [20], is defined by

$$
\phi\left(x^{i}, x^{j}, x^{k}\right)=q^{\bar{i}(\bar{j}+\bar{k}-\overline{j+k}) / \ell}
$$

where $\bar{i}$ denotes the least non-negative residue of $i$ modulo $\ell$, and $q$ a primitive $\ell$-th root of unity. Thus, $\omega_{C}$ is cohomologous to $\phi^{a}$ for some non-negative integer $0 \leq a \leq \ell-1$, and

$$
1=\prod_{r=1}^{\ell-1} q^{a(r+1-\overline{r+1}) / \ell}=q^{a} .
$$

This implies $a=0$ and so $\omega_{C}$ is a coboundary. This completes the proof of the lemma.

Now we can proof Theorem 4.1

Proof Theorem 4.1. By $\left[23\right.$, Cor. 3.6], $D^{\omega}(G)$ is spanned by the set $\Gamma^{\omega}$ of group-like elements when $D^{\omega}(G)$ is commutative. In particular, $\Gamma^{\omega}$ is a finite abelian group which fits into an exact sequence of abelian groups

$$
1 \rightarrow \hat{G} \rightarrow \Gamma^{\omega} \rightarrow G \rightarrow 1
$$

determined by $G$ and $\omega$. Moreover, $D^{\omega}(G)$ is isomorphic to $H\left(\Gamma^{\omega}, \omega^{\prime}\right)$ as quasibialgebras, where $\omega^{\prime} \in Z^{3}\left(\Gamma^{\omega}, \mathbb{C}^{\times}\right)$is the inflation of $\omega^{-1}$ along the above map $\Gamma^{\omega} \rightarrow G$ [23, Sect. 9]. The braiding of $\operatorname{Rep}\left(D^{\omega}(G)\right)$ determines an EM 3-cocycle $\left(\omega^{\prime}, c\right)$ of $\Gamma^{\omega}$. By Lemma 4.5, the existence of a vanishing total indicator if, and only if, $\omega^{\prime}$ is a non-trivial 3-cocycle of $\Gamma^{\omega}$. This is equivalent to that $H\left(\Gamma^{\omega}, \omega^{\prime}\right)$, or equivalently $D^{\omega}(G)$, is a genuine quasi-Hopf algebra.

\section{TAmbara-Yamagami CATEgories}

In this section, we study the positivity of total indicators for integral TambaraYamagami categories associated with elementary p-groups [31]. By [8], these fusion categories are monoidally equivalent to the categories of representations of semisimple quasi-Hopf algebras. In [32], a necessary and sufficient condition is obtained for an integral Tambara-Yamagami category admitting a fibration, i.e. it is monoidally equivalent to the category of representations of a semisimple Hopf algebra. By Corollary 3.5, positivity of total indicators is a necessary condition for the existence of a fibration, but not sufficient in general. We will demonstrate the sufficiency of positivity of total indicators for these integral Tambara-Yamagami categories in this section.

Let $A$ be a finite abelian group. Tambara and Yamagami [31] classified fusion categories with a complete set of simple objects $A \sqcup\{m\}$ satisfying the fusion rules

$$
a \otimes b \cong a b, a \otimes m \cong m \cong m \otimes a, m \otimes m \cong \bigoplus_{x \in A} x \quad(a, b \in A) .
$$

They also showed that such categories are parametrized by pairs $(\chi, \tau)$ where $\chi$ is a non-degenerate symmetric bicharacter of $A$, and $\tau$ is a square root of $|A|^{-1}$. The corresponding fusion category, denoted by $\mathcal{T} \mathcal{Y}(A, \chi, \tau)$, can be described more precisely in the following definition. 
Definition 5.1. $\mathcal{T Y}(A, \chi, \tau)$ is a skeletal fusion category over $\mathbb{C}$ with the set of simple objects $S:=A \sqcup\{m\}$. Hom-sets between elements of $S$ are given by

$$
\operatorname{Hom}\left(s, s^{\prime}\right)= \begin{cases}\mathbb{C} & \text { if } s=s^{\prime} \\ 0 & \text { otherwise }\end{cases}
$$

with $\operatorname{id}_{s}=1 \in \mathbb{C}$. The compositions of morphisms are obvious one. Tensor products of elements of $S$ are given by (5.1) (but with $\cong$ replaced by $=$ ). The unit object $\mathbf{1}$ is strict and equal to the identity $e \in A$. The associativity constraint $\Phi$ is determined by

$$
\begin{aligned}
& \Phi_{a, m, b}=\chi(a, b) \operatorname{id}_{m}: m \rightarrow m, \\
& \Phi_{m, a, m}=\left(\chi(a, x) \delta_{x, y} \operatorname{id}_{x}\right)_{x, y}: \bigoplus_{x \in A} x \rightarrow \bigoplus_{y \in A} y, \\
& \Phi_{m, m, m}=\left(\tau \chi(x, y)^{-1} \operatorname{id}_{m}\right)_{x, y}: \bigoplus_{x \in A} m \rightarrow \bigoplus_{y \in A} m,
\end{aligned}
$$

where $a, b \in A$, and the other $\Phi_{s, t, u}(s, t, u \in S)$ are identity morphisms. Duality of $\mathcal{T Y}(A, \chi, \tau)$ is described as follows: For $a \in A, a^{*}:=a^{-1}$ with $\mathrm{ev}_{a}, \mathrm{db}_{a}$ being identity $\mathrm{id}_{e}$. For the object $m, m^{*}:=m$ with morphisms $\mathrm{ev}_{m}=\tau^{-1} \pi: m \otimes m \rightarrow \mathbf{1}$ and $\mathrm{db}_{m}=\iota: \mathbf{1} \rightarrow m \otimes m$ where $\pi: m \otimes m \rightarrow \mathbf{1}$ and $\iota: \mathbf{1} \rightarrow m \otimes m$ are respectively the canonical projection and embedding satisfying $\pi \iota=\mathrm{id}_{1}$

Since the Frobenius-Perron dimension of $\mathcal{T Y}(A, \chi, \tau)$ is an integer, $\mathcal{T Y}(A, \chi, \tau)$ is pseudo-unitary by [8]. In particular, there exists a unique spherical pivotal structure $j$ for which the pivotal dimension $d(V)$ of an object $V$ is equal to its FrobeniusPerron dimension, i.e.

$$
d(a)=1 \quad \text { for } \quad a \in A, \quad \text { and } \quad d(m)=\sqrt{|A|} .
$$

On can verify directly that $j_{a}=\mathrm{id}_{a}$ for $a \in A$, and $j_{m}=\operatorname{sgn}(\tau) \mathrm{id}_{m}$, where $\operatorname{sgn}(\tau)$ means the sign of the real number $\tau$. We will always assume $\mathcal{T Y}(A, \chi, \tau)$ is a spherical fusion category relative to this canonical pivotal structure $j$.

By [31], two Tambara-Yamagami categories $\mathcal{T Y}(A, \chi, \tau)$ and $\mathcal{T Y}\left(A^{\prime}, \chi^{\prime}, \tau^{\prime}\right)$ are monoidally equivalent if, and only if, $\tau=\tau^{\prime}$ and there exists a group isomorphism $\sigma: A \rightarrow A^{\prime}$ satisfying $\chi(a, b)=\chi^{\prime}(\sigma(a), \sigma(b))$ for all $a, b \in A$ (i.e. $(A, \chi)$ and $\left(A^{\prime}, \chi^{\prime}\right)$ are isometric).

We now turn to the case of elementary $p$-groups $V$ of order $p^{r}$, where $p$ is a prime. Then $V$ is an $r$-dimensional vector space over the finite field $\mathbb{F}_{p}$ of order $p$. A non-degenerate symmetric bilinear form $B: V \times V \rightarrow \mathbb{F}_{p}$ on $V$ determines a non-degenerate symmetric bicharacter $\chi_{B}$ defined by $\chi_{B}(a, b)=\exp (2 \pi i B(a, b))$; moreover, the assignment $B \rightarrow \chi_{B}$ is a one-to-one correspondence. Two bilinear

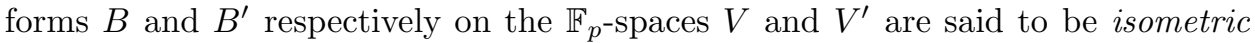

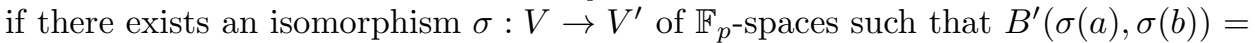
$B(a, b)$ for all $a, b \in V_{1}$. It is easy to see that two bilinear forms $B, B^{\prime}$ are isometric if, and only if, $\chi_{B}, \chi_{B^{\prime}}$ are isometric bicharacters. Moreover, any bilinear form $B$ on a $\mathbb{F}_{p}$-space is uniquely determined by its Gram matrix $\left[B\left(v_{i}, v_{j}\right)\right]_{i j}$ relative to a basis $\left\{v_{i}\right\}_{i}$ of $V$. In particular, we will denote by $B_{0}$ the bilinear form on $\mathbb{F}_{p}^{r}$ whose Gram matrix relative to the standard basis is the identity

Remark 5.2. The Tambara-Yamagami category $\mathcal{T Y}(A, \chi, \tau)$ is integral if, and only if, $d(m)=\sqrt{|A|}$ is an integer, or equivalently $|A|$ is a square. In this case, by [8], the fusion category is monoidally equivalent the representation category of 
a semisimple quasi-Hopf algebra over $\mathbb{C}$. If $V$ is an elementary $p$-group of order $p^{r}$, then $\mathcal{T} \mathcal{Y}(V, \chi, \tau)$ is integral if, and only if, $r$ is even.

5.1. Characteristic two. We will show in this subsection that a Tambara-Yamagami category associated with an elementary 2-group of square order admits a fibration if, and only if, all its total indicators are positive.

Recall that all the non-degenerate alternating bilinear forms on $V=\mathbb{F}_{2}^{r}$ with $r$ even are isometric. Any non-degenerate symmetric bilinear form on $V$, which is not alternating, is isometric to $B_{0}$. In particular, there are exactly two isometric classes of non-degenerate symmetric bilinear on $\mathbb{F}_{2}^{r}$ when $r$ is even. For odd $r$, every non-degenerate symmetric bilinear form is isometric to $B_{0}$ (see, for example, [1]).

Using this classification of symmetric bilinear forms, the indicators of the object $m$ in these Tambara-Yamagami categories have been obtained by Shimizu [30, Thm. 6.3]: For any non-degenerate symmetric bilinear form $B$ on $\mathbb{F}_{2}^{r}$, the $n$-th indicator $\nu_{n}(m)$ of the simple object $m$ in $\mathcal{T Y}\left(\mathbb{F}_{2}^{r}, \chi_{B}, \tau\right)$ is zero if $n$ odd. Moreover,

(i) if $B$ is not alternating, then

$$
\nu_{2 k}(m)=\operatorname{sgn}(\tau)^{k}\left(\frac{1+i}{\sqrt{2}}\right)^{r k}\left(\frac{1+i^{-k}}{\sqrt{2}}\right)^{r} .
$$

(ii) If $r$ is even and $B$ is alternating, then

$$
\nu_{2 k}(m)= \begin{cases}\operatorname{sgn}(\tau) & \text { if } k \text { is odd, } \\ 2^{r / 2} & \text { if } k \text { is even. }\end{cases}
$$

We can now compute the total indicators for the integral Tambara-Yamagami categories associated with an elementary 2-group of square order.

Proposition 5.3. Let $V=\mathbb{F}_{2}^{2 \ell}, B$ a non-degenerate symmetric bilinear form on $V, \tau$ a square root of $|V|^{-1}$, and $\mathcal{C}=\mathcal{T Y}\left(V, \chi_{B}, \tau\right)$.

(i) If $B$ is not alternating, then $\operatorname{FSexp}(\mathcal{C})=8$ and $\bar{\nu}(m)=2 \operatorname{sgn}(\tau)+2^{\ell}$.

(ii) If $B$ is alternating, then $\operatorname{FSexp}(\mathcal{C})=4$ and $\bar{\nu}(m)=\operatorname{sgn}(\tau)+2^{\ell}$.

In particular, $\bar{\nu}(m)=0$ if, and only if, $B$ is not alternating, $\ell=1$ and $\operatorname{sgn}(\tau)=-1$.

Proof. Note that for all $a \in V, \nu_{n}(a)=1=d(a)$ if $n$ is even, and 0 otherwise.

(i) If $B$ is not alternating, then, by (5.2), we have

$$
\nu_{2}(m)=\operatorname{sgn}(\tau), \quad \nu_{4}(m)=0, \quad \nu_{6}(m)=\operatorname{sgn}(\tau), \quad \nu_{8}(m)=2^{\ell}=d(m) .
$$

Therefore, $\operatorname{FSexp}(\mathcal{C})=8$ and $\bar{\nu}(m)=\sum_{k=1}^{4} \nu_{2 k}(m)=2 \operatorname{sgn}(\tau)+2^{\ell}$.

(i) If $B$ is alternating, then, by (5.3), we have

$$
\nu_{2}(m)=\operatorname{sgn}(\tau) \quad \text { and } \quad \nu_{4}(m)=2^{\ell}=d(m) .
$$

Therefore, $\operatorname{FSexp}(\mathcal{C})=4$ and $\bar{\nu}(m)=\sum_{k=1}^{2} \nu_{2 k}(m)=\operatorname{sgn}(\tau)+2^{\ell}$.

The last statement follows directly from statement (i) and (ii).

Corollary 5.4. Let $\mathcal{C}$ be a Tambara-Yamagami category associated with an elementary 2-group $V$ of order $2^{2 \ell}$. Then $\mathcal{C}$ is monoidally equivalent to $\operatorname{Rep}(H)$ for some semisimple Hopf algebra $H$ if, and only if, all its total indicators are positive.

Proof. By [32, Prop 5.5], $\mathcal{C}$ has a fibration if, and only if, $\mathcal{C}$ is not monoidally equivalent to $\mathcal{T Y}\left(\mathbb{F}_{2}^{2}, B_{0},-2\right)$. It follows from Proposition 5.3 that the simple object $m$ in $\mathcal{C}$ satisfies $\bar{\nu}(m)>0$ if, and only if, $\mathcal{C}$ is not monoidally equivalent to $\mathcal{T Y}\left(\mathbb{F}_{2}^{2}, B_{0},-2\right)$. This proves the corollary. 
5.2. Odd characteristic. In contrast to the characteristic two case, positivity of total indicators may not be sufficient for the existence of fibration of an integral Tambara-Yamagami category associated with an elementary $p$-group for odd $p$. We will demonstrate this fact by computing the total indicators. By [29, Ch. 4], there are exactly two isometric classes of non-degenerate symmetric bilinear form on an $r$-dimensional $\mathbb{F}_{p}$-space. They are represented by $B_{0}$ and $B_{1}$ whose Gram matrix relative to the standard basis is given by

$$
\left[\begin{array}{c|c}
I_{r-1} & 0 \\
\hline 0 & u
\end{array}\right]
$$

where $u$ can be any fixed quadratic nonresidue in $\mathbb{F}_{p}$, and $I_{r-1}$ denotes the identity matrix of rank $r-1$. In particular, a non-degenerate symmetric bilinear form $B$ on $\mathbb{F}_{p}^{r}$ is determined by its discriminant $\operatorname{det}(B)$ in $\mathbb{F}_{p}^{\times} /\left(\mathbb{F}_{p}^{\times}\right)^{2}$, where

$$
\operatorname{det} B=\operatorname{det}\left(\left[B\left(e_{i}, e_{j}\right)\right]_{i j}\right) \in \mathbb{F}_{p}^{\times} .
$$

Therefore, the isometric class of a bilinear form $B$ is uniquely determined by the Legendre symbol $\left(\frac{\operatorname{det} B}{p}\right)$ which is 1 if $\operatorname{det} B$ is a quadratic residue, and -1 otherwise. We can now compute the total indicators using the following formula obtained by Shimizu [30, Thm. 6.1]: The $n$-th indicator of $m$ in $\mathcal{T} \mathcal{Y}\left(\mathbb{F}_{p}^{r}, \chi_{B}, \tau\right)$ for any nondegenerate symmetric bilinear form $B$ on $\mathbb{F}_{p}^{r}$ is given by

$$
\nu_{n}(m)= \begin{cases}0 & \text { if } n \text { is odd, } \\ \operatorname{sgn}(\tau)^{k} \varepsilon_{p}^{r(k+1)}\left(\frac{-k}{p}\right)^{r}\left(\frac{-2}{p}\right)^{r(k+1)}\left(\frac{\operatorname{det} B}{p}\right)^{k+1} & \text { if } n=2 k \text { and } p \nmid k, \\ \operatorname{sgn}(\tau)^{k} \varepsilon_{p}^{r k}\left(\frac{-2}{p}\right)^{r k}\left(\frac{\operatorname{det} B}{p}\right)^{k} \sqrt{p^{r}} & \text { if } n=2 k \text { and } p \mid k,\end{cases}
$$

where $\varepsilon_{p}=\sqrt{\left(\frac{-1}{p}\right)}$.

Proposition 5.5. Let $V=\mathbb{F}_{p}^{2 \ell}, \chi_{B}$ the bicharacter associated with a non-degenerate bilinear form $B$ on $V, \tau$ a square root of $|V|^{-1}$, and $\mathcal{C}=\mathcal{T} \mathcal{Y}\left(V, \chi_{B}, \tau\right)$.

(i) If $\left(\frac{-1}{p}\right)^{\ell}\left(\frac{\operatorname{det} B}{p}\right) \operatorname{sgn}(\tau)=1$, then $\operatorname{FSexp}(\mathcal{C})=2 p$ and

$$
\bar{\nu}(m)=p^{\ell}+(p-1) \operatorname{sgn}(\tau) .
$$

(ii) If $\left(\frac{-1}{p}\right)^{\ell}\left(\frac{\operatorname{det} B}{p}\right) \operatorname{sgn}(\tau)=-1$, then $\operatorname{FSexp}(\mathcal{C})=4 p$ and $\bar{\nu}(m)=0$.

Proof. By [30, Thm. 3.2], $\nu_{n}(a)=\delta_{n a, 0}$ for $a \in V$. Therefore, $p \mid \operatorname{FSexp}(\mathcal{C})$. To determine $\operatorname{FSexp}(\mathcal{C})$, it is enough to consider the values $\nu_{n}(m)$ with $n=2 k$ and $p \mid k$ by virtue of (5.4). Note that

$$
\varepsilon_{p}^{2 \ell}\left(\frac{\operatorname{det} B}{p}\right)=\left(\frac{-1}{p}\right)^{\ell}\left(\frac{\operatorname{det} B}{p}\right)=\epsilon \operatorname{sgn}(\tau)
$$

for some $\epsilon= \pm 1$. Therefore, (5.4) becomes

$$
\nu_{n}(m)= \begin{cases}0 & \text { if } n \text { is odd } \\ \operatorname{sgn}(\tau) \epsilon^{k+1} & \text { if } n=2 k \text { and } p \nmid k, \\ \epsilon^{k} p^{\ell} & \text { if } n=2 k \text { and } p \mid k,\end{cases}
$$


If $\epsilon=1$, then $\nu_{2 p}(m)=p^{\ell}=d(m)$ and so $\operatorname{FSexp}(\mathcal{C})=2 p$. Moreover,

$$
\bar{\nu}(m)=p^{\ell}+\sum_{k=1}^{p-1} \nu_{2 k}(m)=p^{\ell}+\operatorname{sgn}(\tau)(p-1) .
$$

If $\epsilon=-1$, then $\nu_{2 p}(m)=-p^{\ell}$ and $\nu_{4 p}(m)=p^{\ell}$. Thus, $\operatorname{FSexp}(\mathcal{C})=4 p$ and

$$
\bar{\nu}(m)=\sum_{k=1}^{2 p} \nu_{2 k}(m)=0 .
$$

Corollary 5.6. Let $B$ be a non-degenerate symmetric bilinear form on $V=\mathbb{F}_{p}^{2 \ell}$ and $\tau= \pm p^{-\ell}$. Then $\mathcal{C}=\mathcal{T} \mathcal{Y}\left(V, \chi_{B}, \tau\right)$ admits a fibration if, and only if, $\bar{\nu}(s) \geq d(s)$ for all simple object $s \in \mathcal{C}$.

Proof. By the preceding proposition, for $s \in V, \bar{\nu}(s) \geq 2>d(s)$. In particular, the inequality holds for all $s \in V$ automatically. Therefore, we only need to consider the simple object $m$.

By [32, Prop. 4.1], $\mathcal{C}$ admits a fibration if, and only if, $\tau=p^{-\ell}$ and $B$ is hyperbolic, i.e. the Gram matrix of $B$ relative to some basis of $\mathbb{F}_{p}^{2 \ell}$ is of the form $\left[\begin{array}{c|c}0 & I_{\ell} \\ \hline I_{\ell} & 0\end{array}\right]$, or equivalently, $\left(\frac{\operatorname{det} B}{p}\right)=\left(\frac{-1}{p}\right)^{\ell}$.

If $\mathcal{C}$ admits a fibration, then $\left(\frac{\operatorname{det} B}{p}\right)\left(\frac{-1}{p}\right)^{\ell}=1=\operatorname{sgn}(\tau)$ and so $\bar{\nu}(m)=p^{\ell}+p-1>$ $p^{\ell}=d(m)$ by Proposition 5.5. Conversely, if $\bar{\nu}(m) \geq p^{\ell}$, then, by Proposition 5.5, $\operatorname{sgn}(\tau)=1$ and $\left(\frac{\operatorname{det} B}{p}\right)\left(\frac{-1}{p}\right)^{\ell}=1$, or equivalently $\left(\frac{\operatorname{det} B}{p}\right)=\left(\frac{-1}{p}\right)^{\ell}$. Therefore, $\mathcal{C}$ admits a fibration by the preceding paragraph.

Remark 5.7. The corollary implies that there exists a genuine semisimple quasiHopf algebra of which the total indicators of its representations are all positive. Let $B$ be the bilinear form on $\mathbb{F}_{p}^{2 \ell}$ whose Gram matrix relative to the standard basis is $\left[\begin{array}{c|c}I_{2 \ell-1} & 0 \\ \hline 0 & (-1)^{\ell+1}\end{array}\right]$. Then $\left(\frac{\operatorname{det} B}{p}\right)\left(\frac{-1}{p}\right)^{\ell}=-1$. If we take $\operatorname{sgn}(\tau)=-1$ or $\tau=-p^{\ell}$, then, by Corollary 5.6 and Proposition $5.5, \mathcal{T} \mathcal{Y}\left(\mathbb{F}_{p}^{2 \ell}, \chi_{B}, \tau\right)$ is monoidally equivalent to $\operatorname{Rep}(H)$ of a genuine quasi-Hopf algebra $H$ with positive total indicators. Therefore, in general, the existence of vanishing total indicators is not a necessary condition for a semisimple quasi-Hopf algebra being genuine.

\section{REFERENCES}

[1] A. A. Albert, Symmetric and alternate matrices in an arbitrary field. I, Trans. Amer. Math. Soc. 43 (1938), no. 3, 386-436. MR 1501952

[2] B. Bakalov and A. Kirillov Jr., Lectures on tensor categories and modular functors. University Lecture Series, 21. American Mathematical Society, Providence, RI, 2001.

[3] P. Bantay, The Frobenius-Schur indicator in conformal field theory. Phys. Lett. B, 394(12):87-88, 1997.

[4] S. Burciu, On some representations of the Drinfeld double. J. Algebra 296 (2006), no. 2, 480-504.

[5] R. Dijkgraaf, V. Pasquier and P. Roche, Quasi Hopf algebras, group cohomology and orbifold models. Nuclear Phys. B Proc. Suppl. 18B (1990), 60-72.

[6] P. Etingof and S. Gelaki, On the exponent of finite-dimensional Hopf algebras. Math. Res. Lett. 6 (1999), no. 2, 131-140.

[7] P. Etingof and S. Gelaki, On families of triangular Hopf algebras. Int. Math. Res. Not. (2002), no. $14,757-768$. 
[8] P. Etingof, D. Nikshych and V. Ostrik, On fusion categories. Ann. of Math. (2) 162 (2005), no. 2, 581-642.

[9] C. Goff, G. Mason and S.-H. Ng, On the gauge equivalence of twisted quantum doubles of elementary abelian and extra-special 2-groups. J. Algebra 312 (2007), no. 2, 849-875.

[10] A. Joyal and R. Street, Braided monoidal categories, Macquarie Math. Reports No. 850067, Dec 1985; Revised No. 860081, Nov. 1986.

[11] Y. Kashina, On the order of the antipode of Hopf algebras in ${ }_{H}^{H}$ YD. Comm. Algebra, 27 (1999), no. 3, 1261-1273.

[12] Y. Kashina, A generalized power map for Hopf algebras. In Hopf algebras and quantum groups (Brussels, 1998), volume 209 of Lecture Notes in Pure and Appl. Math., pages 159175. Dekker, New York, 2000.

[13] Y. Kashina, Y. Sommerhäuser and Y. Zhu, On higher Frobenius-Schur indicators. Mem. Amer. Math. Soc. 181 (2006), no. 855, viii+65 pp.

[14] C. Kassel, Quantum groups. Springer-Verlag, New York, 1995.

[15] R. G. Larson and D. E. Radford, Finite-dimensional cosemisimple Hopf algebras in characteristic 0 are semisimple. J. Algebra, 117 (1988), no. 2, 267-289.

[16] V. Linchenko and S. Montgomery, A Frobenius-Schur theorem for Hopf algebras. Special issue dedicated to Klaus Roggenkamp on the occasion of his 60th birthday, Algebr. Represent. Theory 3 (2000), no. 4, 347-355.

[17] S. MacLane, Cohomology theory of Abelian groups, Proceedings of the International Congress of Mathematicians, Cambridge, Mass., 1950, vol. 2, Amer. Math. Soc., Providence, R. I., 1952, pp. 8-14.

[18] A. Masuoka, Semisimple Hopf algebras of dimension 6, 8. Israel J. Math. 92 (1995), no. 1-3, $361-373$.

[19] S. Montgomery. Hopf algebras and their actions on rings, volume 82 of CBMS Regional Conference Series in Mathematics. Published for the Conference Board of the Mathematical Sciences, Washington, DC, 1993.

[20] G. Moore and N. Seiberg, Classical and quantum conformal field theory, Comm. Math. Phys. 123 (1989), no. 2, 177-254.

[21] M. Müger, Tensor categories: a selective guided tour. Rev. Un. Mat. Argentina 51 (2010), no. 1, 95-163.

[22] M. Müger, From subfactors to categories and topology. II. The quantum double of tensor categories and subfactors, J. Pure Appl. Algebra 180 (2003), no. 1-2, 159-219.

[23] G. Mason and S.-H. Ng, Group cohomology and gauge equivalence of some twisted quantum doubles. Trans. Amer. Math. Soc. 353 (2001), no. 9, 3465-3509.

[24] G. Mason and S.-H. Ng, Central invariants and Frobenius-Schur indicators for semisimple quasi-Hopf algebras. Adv. Math., 190 (2005), no. 1,161-195.

[25] S.-H. Ng and P. Schauenburg, Central invariants and higher indicators for semisimple quasiHopf algebras. Trans. Amer. Math. Soc. 360 (2008), no. 4, 1839-1860.

[26] S.-H. Ng and P. Schauenburg, Frobenius-Schur indicators and exponents of spherical categories. Adv. Math., 211 (2007), no. 1, 34-71.

[27] S.-H. Ng and P. Schauenburg, Higher Frobenius-Schur indicators for pivotal categories. Contemporary Math., 441, 2007, 63-90.

[28] S.-H. Ng and P. Schauenburg, Congruence subgroups and generalized Frobenius-Schur indicators. Comm. Math. Phys. 300 (2010), no. 1, 1-46.

[29] J.-P. Serre, A course in arithmetic, Springer-Verlag, New York, 1973, Translated from the French, Graduate Texts in Mathematics, No. 7.

[30] K. Shimizu, Frobenius-Schur indicators in Tambara-Yamagami categories. J. Algebra 332 (2011), 543-564.

[31] D. Tambara and S. Yamagami, Tensor categories with fusion rules of self-duality for finite abelian groups. J. Algebra 209 (1998), no. 2, 692-707.

[32] D. Tambara, Representations of tensor categories with fusion rules of self-duality for abelian groups. Israel J. Math. 118 (2000), 29-60.

[33] V. G. Turaev. Quantum invariants of knots and 3-manifolds. volume 18 of de Gruyter Studies in Mathematics. Walter de Gruyter \& Co., Berlin, 1994.

[34] C. Vafa, Toward classification of conformal theories. Phys. Lett. B 206 (1988), no. 3, 421-426. 
Department of Mathematics, Nanjing University, Nanjing 210093, China

E-mail address: gxliu@nju.edu.cn

Department of Mathematics, Iowa State University, Ames, IA 50011, USA

E-mail address: rng@iastate.edu

Current address: Department of Mathematics, Cornell University, Ithaca, NY 14850, USA 